lives of 10,33 , and $112 \mathrm{~min}$. after the shorter-lived activities decayed out. The 33- and 112-min. activities were shown to be associated with the expected $\mathrm{Cl}^{34}$ and $\mathrm{F}^{18}\left(\mathrm{O}^{18}(p, n) \mathrm{F}^{18}\right)$; the source of the 10-min. activity was puzzling until chemical and absorption tests showed it to be the previously known ${ }^{5} \mathrm{~N}^{13}$. The only conceivable reaction which would lead to this activity in the targets used is $\mathrm{O}^{16}(p, \alpha) \mathrm{N}^{13}$. This reaction, as well as the $\mathrm{S}^{34}(p, n) \mathrm{Cl}^{34}$ reaction, seems not to have been reported before.

Following a bombardment the target crystals were dissolved in water, $\mathrm{Cl}^{-}, \mathrm{ClO}_{3}^{-}$, and $\mathrm{ClO}_{4}^{-}$carriers were added, as well as $\mathrm{F}^{-}$ hold-back carrier, and a $\mathrm{Cl}^{-}$fraction precipitated with excess $\mathrm{Ag}^{+}$. The filtrate was treated with $\mathrm{SO}_{2}$ or $\mathrm{NO}_{2}^{-}$to reduce higher valent states, excepting $\mathrm{ClO}_{4}^{-}$, to $\mathrm{Cl}^{-}$, and a second $\mathrm{AgCl}$ precipitate removed. Finally, the $\mathrm{ClO}_{4}^{-}$was precipitated with methylene blue. ${ }^{6}$ All precipitates were filtered onto paper disks, dried, and mounted for counting ${ }^{7}$ in a reproducible geometry under a GeigerMüeller counter and scale-of-64, the 2.5- and 5.1-Mev positrons ${ }^{5}$ being used for the detection. Typical results are shown in Table I.

TABLE I. Corrected activities of $\mathrm{Cl}$ fractions from $\mathrm{S}^{\boldsymbol{S}}(p, n) \mathrm{Cl}^{34}$ reaction.

\begin{tabular}{lcr} 
Fraction & $\mathrm{Na}_{2} \mathrm{SO}_{4}$ & $\mathrm{~K}_{2} \mathrm{~S}_{2} \mathrm{O}_{8}$ \\
$\mathrm{Cl}^{-}$ & $13743 \pm 115$ & $1047 \pm 24$ \\
$\mathrm{ClO}^{-1}$ & $22 \pm 3$ & $0 \pm 2$ \\
$\mathrm{ClO}_{4}-$ & $40 \pm 3$ & $9 \pm 2$ \\
\hline
\end{tabular}

a Error includes only standard counting error.

These experiments indicate that essentially all of the $\mathrm{Cl}^{34}$ activity appears as $\mathrm{Cl}^{-}$or in a form rapidly exchangeable with $\mathrm{Cl}^{-}$; thermal exchange between $\mathrm{Cl}^{-}$and $\mathrm{ClO}_{2}, \mathrm{ClO}_{3}^{-}$, and $\mathrm{ClO}_{4}^{-}$under the given conditions has been shown ${ }^{8-10}$ to be slow, whereas $\mathrm{Cl}^{-}$ and $\mathrm{Cl}_{2}$ are known ${ }^{11}$ to undergo rapid exchange. It is indeterminate whether the $\mathrm{Cl}^{-}$exists in the crystal lattace as such or arises as a result of dissolving the crystals. The hot chlorine atom may be ejected as a neutral atom or positive ion and take up electrons before it can react with the oxygen in the crystal. Similar results have been reported ${ }^{8}$ for the Szilard-Chalmers reaction on $\mathrm{ClO}_{4}^{-}$.

An attempt was made to determine the chemical state of the $\mathrm{N}^{13}$, but no quantitative results can be given. The addition of $\mathrm{NH}_{4}{ }^{+}$carrier to the solution (chemically freed from $\mathrm{Cl}^{34}$ and $\mathrm{F}^{18}$ activities), followed by its precipitation with Nessler's reagent, carried down a moderately large, but unreproducible, fraction of the $\mathrm{N}^{13}$ activity. A Kjeldahl reaction experiment indicated higher oxidation states were present. The $\mathrm{N}^{13}$ does not exist appreciably in any volatile form in the solution inasmuch as little activity was lost when the aqueous solution was evaporated to dryness. Since it was important to know whether exchange would take place between certain oxidation states in the time required for the experiments, the exchange of $\mathrm{NH}_{4}{ }^{+}$and $\mathrm{NO}_{2}^{-}$was investigated briefly. In solutions $0.23 f$ in both $\mathrm{NH}_{4}^{+}$and $\mathrm{NO}_{2}^{-}$, and $0.012 f$ in $\mathrm{H}^{+}$, $3.3 \pm 2.6$ percent exchange was found after $92 \mathrm{~min}$. at room temperature, separation being achieved by the use of Nessler's reagent.

1 W. F. Libby, J. Am. Chem. Soc. 69, 2523 (1947)

2 See, for example, A. H. W. Aten, Jr., Phys. Rev. 71, 641 (1947); Sherr Muether, and White, Phys. Rev. 75, 282 (1949).

3 Aten (see reference 2 ) found that the radiophosphorus produced by $(n, p)$ or $(n, \alpha)$ reactions with radium-beryllium neutrons on inorganic crystal containing sulfur or chlorine tends to form phosphates in oxygen-rich crystals.

4 We wish to thank Professor J. R. Richardson and the cyclotron crew for their co-operation in arranging the bombardments.

5 G. T. Seaborg and I. Perlman. Rev. Mod. Phys. 20, 585 (1948).

- F. J. Welcher, Organic Analytical Reagents (D. Van Nostrand Company, Inc., New York, 1948), Vol. IV.

7 Background, decay, and coincidence corrections were applied. Activities in the precipitates were shown to be due only to $33-\mathrm{min}$. $\mathrm{Cl}^{34}$ by decay measurements. Final filtrates were counted with a dipping counter and no residual $\mathrm{Cl}^{34}$ activity was found.

8 W. F. Libby, J. Am. Chem. Soc. 62, 1930 (1940).

$\checkmark$ H. Dodgen and H. Taube, J. Am. Chem. Soc. 71, 2501 (1949)

${ }_{10} \mathrm{We}$ confirmed that the exchange between $\mathrm{Cl}^{-}$and $\mathrm{ClO}_{3}{ }^{-}$and between

$\mathrm{Cl}^{-}$and $\mathrm{ClO}_{4}^{-}$is very slow for our experimental conditions.

$1 \mathrm{~F}$. A. Long and A. R. Olson, J. Am. Chem. Soc. 58, 2214 (1936).

\section{Photo-Chemical Reduction of Water by Europium \\ (II) Ion, and the Magnetic Susceptibilities of Europium (II) and (III) Ions}

\author{
David L. Dougr.AS AND Don M. Yost \\ Gates and Crellin Laboratories of Chemistry, California Institute of Technology. \\ Pasadena, California*
}

October 14, 1949

$7 \mathrm{HE}$ slow reaction of europium (II) ion with hydrogen ion or water to.produce hydrogen and europium $(I I I)$ ion has been previously reported..$^{1-4}$ Prior to an investigation of the aqueous chemistry of europium (II) ion, the study of this reaction was undertaken. It was decided to follow the course of the reaction by observing the change in magnetic susceptibility of the reaction mixture, using a Gouy balance. This method was suggested by the aforementioned work of R. A. Cooley. ${ }^{4}$ Preliminary experiments showed that solutions $0.25 \mathrm{~m}$ in europium $(I I)$ chloride and about $0.3 \mathrm{~m}$ in hydrochloric acid were stable over periods of weeks when kept in the dark, but evolved hydrogen and changed susceptibility rapidly when exposed to bright light (either sunlight or a tungsten lamp). One other such system has been reported in the literature. Poterill, Walker, and Weiss investigated the photo-chemical reduction of water by iron (II) ion. ${ }^{5}$

A systematic investigation of this reaction, including the thermal reaction, has been in progress for some time. The apparatus will be described in detail in a future report. It permits the sidewise irradiation, with the $3660 \mathrm{~A}$ line group of a $20-\mathrm{cm}$ mercury arc, of a Gouy tube containing the reaction mixture and an identical tube containing a uranyl oxalate actinometer. Experiments so far have been confined to solutions $0.50 \mathrm{~m}$ in $\mathrm{HCl}, 0.15 \mathrm{~m}$ in $\mathrm{EuCl}_{2}$ and $0.05 \mathrm{~m}, 1.05 \mathrm{~m}$ or $2.05 \mathrm{~m}$ in $\mathrm{KCl}$, the ionic strength in these cases being 1.0, 2.0 and 3.0 respectively. Table I contains a summary of some of the results obtained thus far. It is considered that insufficient data are available to elucidate completely the mechanism and so a discussion of this will be deferred.

TABLE I. Quantum yields of photo-chemical reduction of water by europium (II) ion.*

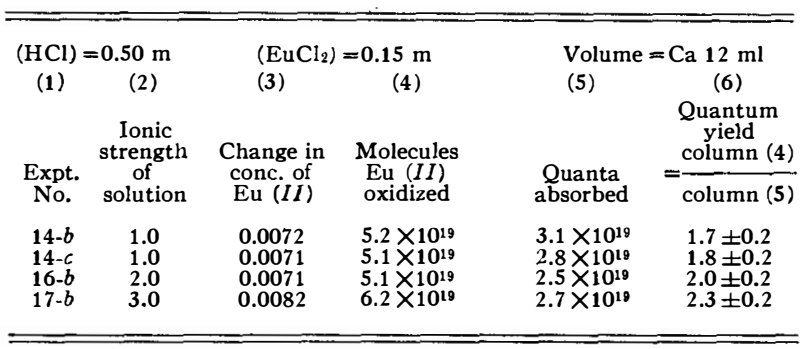

* Activated by the $3660 \mathrm{~A}$ radiation, of which $\mathrm{Eu}(I I)$ is the only absorber in these solutions.

With regard to the analytical method, the literature contains many values of the magnetic susceptibility of europium (III) ion. Yost, Russell, and Garner present a summary of these. ${ }^{6} \mathrm{We}$ have determined the molar susceptibility of europium (III) ion in aqueous solution at $25.0^{\circ} \mathrm{C}$ to be $4.50 \times 10^{3}$ c.g.s. units; this value is independent of the ionic strength of the solution. The magnetic susceptibilities of a number of compounds of europium (II) is reported ${ }^{7.8}$ but no value for the ion in solution appears in the literature. By working in an atmosphere of carbon dioxide when preparing the solutions, and when carrying out analyses, by the method recommended by $\mathrm{H}$. N. McCoy, ${ }^{9}$ we have measured the susceptibility of europium $(I I)$ ion in aqueous solution, over the concentration range $0.3 \mathrm{~m}$ to $0.1 \mathrm{~m}$, with the following result: $\chi_{M}=26.2_{6} \pm 0.1 \times 10^{3}$ c.g.s. $/ g$ ion. The ionic diamagnetic susceptibilities listed by L. F. Bates ${ }^{10}$ were used throughout. This value of $\chi_{M}$ is in good agreement with Van Vleck's ${ }^{11}$ theoretical value of $26.1 \times 10^{3}$ c.g.s./g ion for the isoelectronic gadolinium 
(III) ion. In calculating the amounts of europium present in the two oxidation states the validity of Wiedemann's additivity law was assumed.

The europium used in these researches was a gift of the late Dr. H. N. McCoy and was of greater than 99.9 percent purity as regards other rare earths. Our supply was augmented by a loan of more of the same material by Mrs. Ethel Terry McCoy, for which we are exceedingly grateful.

We wish to thank Professor N. Davidson and Dr. O. Wulf for many helpful discussions and suggestions. We are also very grateful to the Research Corporation for a grant which aided, in part, the work reported here. The junior author (David L. Douglas) is indebted to the Atomic Energy Commission for financial assistance in the form of a fellowship for the year 1948-49.

* Contribution No. 1345.

1 Yost, Russell, and Garner, The Rare Earth Elements and Their Compounds (John Wiley and Sons, Inc., New York, 1947), page 66.

2 Jantsch, Grubitsch, and Lischka, Zeits. f. Electrochemie 43, 293 (1937).

3 F. D. S. Butement. Trans. Faraday Soc. XIIV, 617 (1948).

R. A. Cooley, Ph.D. Thesis, California Institute of Technology, 1941.

5 Poterill, Walker, and Weiss, Proc. Roy Soc. A156, 561 (1936).

- See reference 1, page 14

7 P. W. Selwood, J. Am. Chem. Soc. 55, 4869 (1933)

8 W. Klemm and W. Döll, Zeits. f. anorg. unt allg. Chem. 241, 233 (1939).

- H. N. McCoy, J. Am. Chem. Soc. 61, 2455 (1939).

10 L. F. Bates, Modern Magnelism (Cambridge University Press, London

1947). page 132. Vleck, Electric and Magnetic Susceplibilities (Oxford University Press, Cambridge, 1932), page 243.

\section{Spectra of Disordered Crystals}

\author{
D. F. Hornic
}

Melcalf Research Laboralory, Brown Universily, Providence, Rhode Island October 3, 1949

$\mathrm{T}$ HE spectra of crystals, both electronic and vibrational, have been discussed by a number of authors. ${ }^{1-5}$ All of these discussions have been based on the premise that a crystal is a perfectly ordered system with a clearly defined structure about all interior points. Elementary statistical considerations make it apparent that if alternative structures which do not differ too much in their free energy are possible, and if they are accessible in the sense that the activation energy for the transition to the alternative structure is not too high, a finite fraction of the domains of the crystal will exist with alternative structures at any temperature other than absolute zero. That is, disorder must be very common in crystals at finite temperatures. It is the purpose of this note to point out (a) that the selection rules and character of the spectra cannot be simply obtained from the crystallographic symmetry in such cases and (b) that spectra may be expected to be sensitive to short-range or local order and are therefore a useful complement to $\mathrm{x}$-ray methods which are most sensitive to longrange order.

Disorder has been extensively investigated in alloy structures. ${ }^{6}$ Evidence is also available for disorder in many molecular and ionic crystals, a particularly common type being that in which molecules or ions are located at essentially fixed lattice points but in which the equilibrium orientation of each molecule is not specified. Free rotation would be a particular case of such disorder although it is questionable whether free rotation in a crystal has been definitely demonstrated in even a single case. Examples of such disorder might include crystalline carbon monoxide, the cubic modifications of many nitrates and a variety of crystals involving symmetric molecules or ions. A common feature of disordered phases of this kind is that the crystal symmetry is higher than in the ordered (or less disordered) phase existing at lower temperatures. Several authors have accordingly considered that the effective symmetry for vibrational spectra is also higher, implying more stringent selection rules, absence of splitting of degeneracies, etc. In fact, the symmetry of the Hamiltonian is always reduced in passing to a less ordered phase and we may rather expect that selection rules will be relaxed and degeneracies split; in some cases the spectrum may approach that of a liquid.

Since the spectra of molecules and ions in a crystal differ only very slightly from the spectra of the corresponding gases, they must be only very slightly perturbed by the crystalline environment. In this case the Hamiltonian of the crystal may be written

$$
H=\sum_{i} H_{i}{ }^{0}+H^{\prime}
$$

where $H_{i}{ }^{0}$ is the internal Hamiltonian of the $i$ th molecule and $H^{\prime}$ is the small perturbation due to the crystal. If $H^{\prime}$ is expanded in terms of nearest neighbor interactions, next nearest neighbor interactions, etc., the successive terms decrease rapidly in magnitude since all of the interactions fall off at least as rapidly as the inverse square of the distance and proposition (b) follows immediately, that most of the observed effects are due to local structures. Since in a disordered state a variety of local structures coexist, the symmetry effects may be expected to vanish to a degree depending on the nature of the local structures. In general, one may expect that in a disordered structure the sharp line spectra expected in ordered structures ${ }^{4,5}$ should be broadened.

Such effects appear to have been observed in studies of the vibrational spectra of ammonium and deutero-ammonium halides above (disordered) and beneath (ordered) the $\lambda$-point. ${ }^{7}$ It is hoped that the spectra of crystals may provide a generally useful tool in studying order-disorder transitions of transparent substances and further experimental studies are now in progress.

1 H. Bethe, Ann. d. Physik 3, 133 (1929).

2 S. Bhagavantam and T. Venkatarayudu, Proc. Ind. Acad. Sci. A9, 224 (1939).

${ }^{3}$ R. S. Halford, J. Chem. Phys. 14, 8 (1946); H. Winston and R. S. Halford, ibid. 17, 607 (1949).

D. F. Hornig, J. Chem. Phys. 16, 1063 (1948)

6 A. S. Davydov, J. Exp. Theor. Phys. (U.S.S.R.) 18, 210 (1948).

- F. C. Nix and W. Shockley, Rev. Mod. Phys. 10, 1 (1938).

7 E. L. Wagner and D. F. Hornig, J. Chem. Phys. (to be published); see Phys. Rev. 75, 1282 (1949).

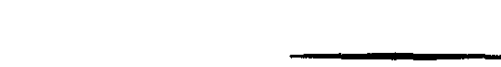

\section{Solubility of Water in Hydrocarbons}

\section{J. H. HiLdEBRAND}

Department of Chemistry, Universily of California, Berkeley, California October 3, 1949

VALUABLE paper bearing this title was published recently by Black, Joris, and Taylor. ${ }^{1}$ They reported the solubility of water in $\mathbf{1 5}$ hydrocarbons and discussed the results in connection with the usual equation for regular solutions, and also with an equation of the Flory-Huggins type designed to take account of discrepancy in molal volumes.

In view of the general importance of being able to predict the solubility of water in any hydrocarbon, I believe that the following method of analyzing and correlating these results will prove useful to many.

The equations for the heat of mixing and the entropy of mixing may be combined to give ${ }^{2}$

$$
R T \ln \left(a_{2} / \varphi_{2}\right)=\mathbf{v}_{2} \varphi_{1}{ }^{2}\left(\delta_{1}-\delta_{2}\right)^{2}+R T \varphi_{1}\left(1-\mathbf{v}_{2} / \mathbf{v}_{1}\right),
$$

where $a$ denotes activity, $\mathrm{v}$ molal volume, $\varphi$ volume fraction, and $\delta$ the "solubility parameter," which is the square root of the energy of vaporization per cc. We shall let subscript 2 stand for water and 1 for the hydrocarbon. The first term on the right is intended to account for the heat, the second the entropy of mixing. The latter should be unusually significant in these solutions because of the relatively small molal volume of water.

One would hardly be surprised if a value of $\delta_{2}$ derived from the heat of vaporization of water would fail to fit these solutions since hydrogen bonds contribute so largely to the cohesion of liquid water but not to the attraction between water and hydrocarbon molecules. It seemed possible, nevertheless, that a uniform even if somewhat artificial solubility parameter for water might serve to 